This item was submitted to Loughborough's Research Repository by the author.

Items in Figshare are protected by copyright, with all rights reserved, unless otherwise indicated.

\title{
Cocrystallization of urea and succinic acid in "Nano-Crystallizer"
}

PLEASE CITE THE PUBLISHED VERSION

https://doi.org/10.1016/j.ces.2020.116082

PUBLISHER

Elsevier BV

VERSION

AM (Accepted Manuscript)

PUBLISHER STATEMENT

This paper was accepted for publication in the journal Chemical Engineering Science and the definitive published version is available at https://doi.org/10.1016/j.ces.2020.116082

\section{LICENCE}

CC BY-NC-ND 4.0

\section{REPOSITORY RECORD}

Yu, Qiushuo, Wanying Jia, Jiaojiao Pu, Yingchen Wang, and Huaiyu Yang. 2020. "Cocrystallization of Urea and Succinic Acid in "nano-crystallizer"'. Loughborough University. https://hdl.handle.net/2134/16803697.v1. 


\title{
Cocrystallization of Urea and Succinic Acid in "Nano- Crystallizer"
}

\author{
Qiushuo $\mathrm{Yu}^{\dagger *}$, Wanying Jia', Jiaojiao $\mathrm{Pu}^{\dagger}$, Yingchen Wang ${ }^{\dagger}$, Huaiyu Yang ${ }^{\S *}$ \\ 'School of Chemical Engineering, Northwest University, Xi'an, Shaanxi, People's Republic \\ of China \\ $\S$ Department of Chemical Engineering, Loughborough University, Loughborough, UK \\ Corresponding Author: \\ *E-mail: yuqiushuo@nwu.edu.cn (Yu), H.Yang3@lboro.ac.uk (Yang)
}

\begin{abstract}
In the bulk scale of crystallization, urea-succinic acid (U-SA) cocrystals have been reported to only form stable 2:1 U-SA cocrystal, without any metastable 1:1 cocrystal, by slow evaporation and cooling crystallization in an aqueous solvent. In this work, cooling crystallization and evaporation crystallization were applied in nano-crystallizers, i.e. the nano-pores in controlled pore glass (CPG). It is the first time to demonstrate that, with confined solution, it is possible to produce and maintain metastable cocrystals during the slow crystallization process, indicating the strong influences of the confinement on the phase diagram and the thermodynamic properties of the nano-scale cocrystals. The influences of the urea and succinic acid concentration and the pore size of CPG on the polymorphs and melting point of the nanocrystal have been investigated. Further understanding of the mechanism may contribute to new methods for maintaining the unstable materials and discover new forms of pharmaceutical compounds and materials.
\end{abstract}

Key words: Nanocrystal, Cocrystal, Urea, Succinic Acid, Nano-crystallizer 


\section{Introduction}

Confinement crystallization has been reported as crystallization occurs in nano/micro sized droplets (Yang et al., 2015) or in the nano/micro pores (Scherer, 1999), by electro-spraying (Padrela et al., 2014), spray-drying step (Tiago et al., 2013), supercritical fluid (Padrela et al., 2014), or in Nanocellulose (Skorupska et al., 2014), nanoporous siliceous matrices (Ha et al., 2009), in Controlled Pore Glass (CPG) (Jackson and McKenna, 1996). When a solution is confined in a nano/micrometer scale, the confinement redefines the free energy of crystal surface, structure and properties (Vekilov, 2010)(Zabara et al., 2017)(Munekawa et al., 2015). Therefore, confinement attributes to the screening of new polymorphs of active pharmaceutical ingredient (API) (Ha et al., 2004), and can be used to control and manufacture different polymorphs (Jiang et al., 2013) and to facilitate protein crystallization(Chen et al., 2019). The pharmaceuticals under confinement potentially lead to new designs of the drug release and delivery (Charnay et al., 2004), which drive a key preparational step of further applications in individual therapies (Skorupska et al., 2014). Cocrystals of API with a conformer can improve pharmaceutical performance such as solubility, dissolution rate, stability, and hygroscopicity (Chen et al., 2016)(Duggirala et al., 2014)(Gopi et al., 2016)(Kaur et al., 2017)(Duggirala et al., 2016). Crystallization is an essential for manufacturing pharmaceuticals, cocrystallization or nano-scale crystallization (Powell et al., 2016)(Dai et al., 2018)(Coceani et al., 2012) provide new approaches to create new types of materials and to discover new solid forms of pharmaceutically active compounds (James et al., 2012). However, there are only few reports on combining nano-technology with co-crystallization technology, neither the slow crystallization process of the nano-scale co-crystallization was reported.

Urea and succinic acid can form two types of cocrystals. The thermodynamically stable 2:1 urea and succinic acid (U-SA) cocrystal was 
easily obtained by cooling crystallization or evaporation from various solvents (Yao et al., 2014). 1:1 U-SA cocrystal, which is metastable, quickly transforms to $2: 1$ cocrystal in a bulk water environment. The 1:1 cocrystal can be obtained by generating high supersaturation in water, such as spraying drying and freeze drying (Alhalaweh et al., 2010)(Wiedenfeld and Knoch, 1990)(Yu et al., 2019), which separates the water phase and cocrystal phase in a very short time. However, there is no report on producing 1:1 cocrystal in pure water solvent by slow cooling and slow evaporation crystallization in bulk solution. CPG with narrow pore size distribution was obtained after leaching forms a continuous, silica-rich glass with a porous network of specified pore dimensions. CPG has been frequently used to confine substances like hydrogen and helium, inert gases, water and organic liquids. The properties of liquids and solids have been altered under confinement, such as melting point, dissolution and polymorphs (Kanakubo et al., 2006)(Dosseh et al., 2003)(Dwyer et al., 2015)(Ha et al., 2004).

In this work, the CPGs with average pore diameters of 40, 50, 60,100 and 190 $\mathrm{nm}$ were used as templates and the nm-scale pores of the templates acted as nano-crystallizers. Cooling and evaporation crystallization, with 1:1 and 2:1 concentration ratios of urea/SA, were performed in nano-crystallizers, compared with the crystallization experiments in bulk solution. By combining nano-technology and cocrystallization technology, it is the first time revealed that the 1:1 U-SA cocrystal can be obtained by slow evaporation and cooling crystallization, and the metastable 1:1 cocrystals can coexist with water, without transforming to 2:1 cocrystal, during a long period in nano-crystallizers.

\section{Material and methods}

Urea (mass fraction purity $>99.0 \%$ ) and succinic acid (mass fraction purity $>$ $99.0 \%$ ), from Alfa Aesar, were used without further treatments. Deionized water was used. The grain sizes for all the CPGs are in 0.074 and $0.125 \mathrm{~mm}$, with density $0.28 \pm 0.01 \mathrm{~g} \mathrm{~cm}^{-3}$. CPGs, from Prime Synthesis Inc (Nelson, 1995), of $40,50,60,100$ and $190 \mathrm{~nm}$ pore sizes, representing mean pore size of 38.2 
(CPG-40), 52.8 (CPG-50), 62.6 (CPG-60), 100.3 (CPG-100) and $191.0 \mathrm{~nm}$ (CPG-190), have a specific surface area 113, 81, 79, 51, $22 \mathrm{~m}^{2} \mathrm{~g}^{-1}$, respectively.

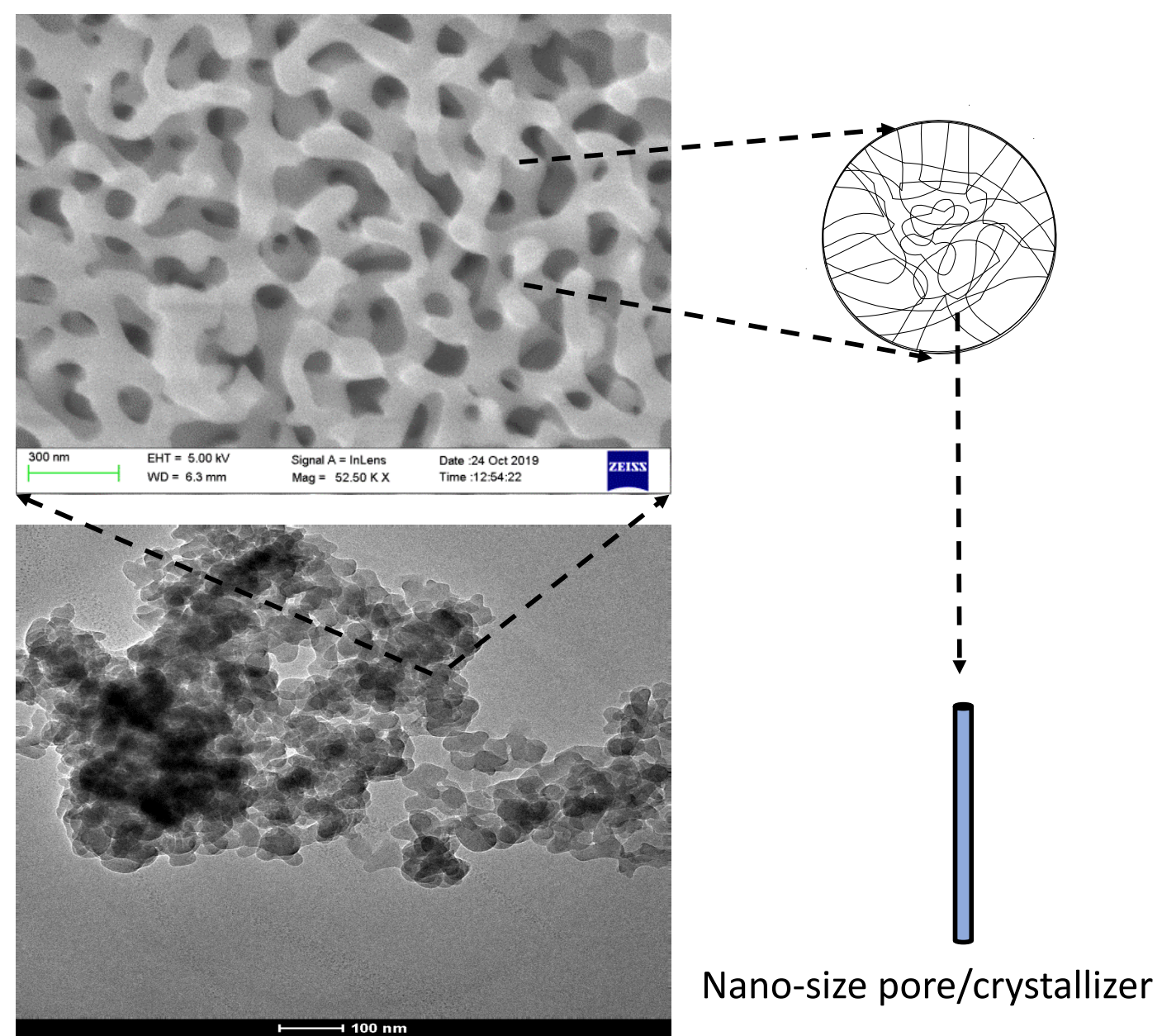

Figure 1. TEM and SEM images of CPGs

Controlled pore glass (CPG) formed by alkali borosilicate glasses with pores with narrow distributions of average pore diameters of 40,50,60,100 and 190 $\mathrm{nm}$ were used. Before crystallization, the CPGs, shown in Figure 1, were submerged in $30 \%$ nitric acid at $100{ }^{\circ} \mathrm{C}$ for $3 \mathrm{~h}$ and then washed with water five times, before drying under a vacuum for $24 \mathrm{~h}$. Urea and succinic solutions, with 2:1 or 1:1 molar ratio between urea and succinic acid, were prepared by dissolving $0.4 \mathrm{~g}$ or $0.8 \mathrm{~g}$ succinic acid and the corresponding amount of urea in $10 \mathrm{~mL}$ water in a $25 \mathrm{~mL}$ vial at room temperature. The solution was dropped on the surface of CPGs and immediately absorbed inside pores of CPGs or the 
CPGs were submerged in the urea and succinic solution to absorb the solution. The remaining solution outside the pores was removed by filtration paper. The pores of CPGs were used as nano-crystallizer (Figure 1) for the cooling and evaporation crystallization in Exp. 1 - 4. Exp. 5 and 6 (as reference experiments) were performed with a larger scale of $10 \mathrm{~mL}$ solution in a $25 \mathrm{~mL}$ vial with the same concentration as in Exp. 1- 4, under the same evaporation and cooling conditions as in Exp. 1-4. The experiments were repeated at least three times at each condition.

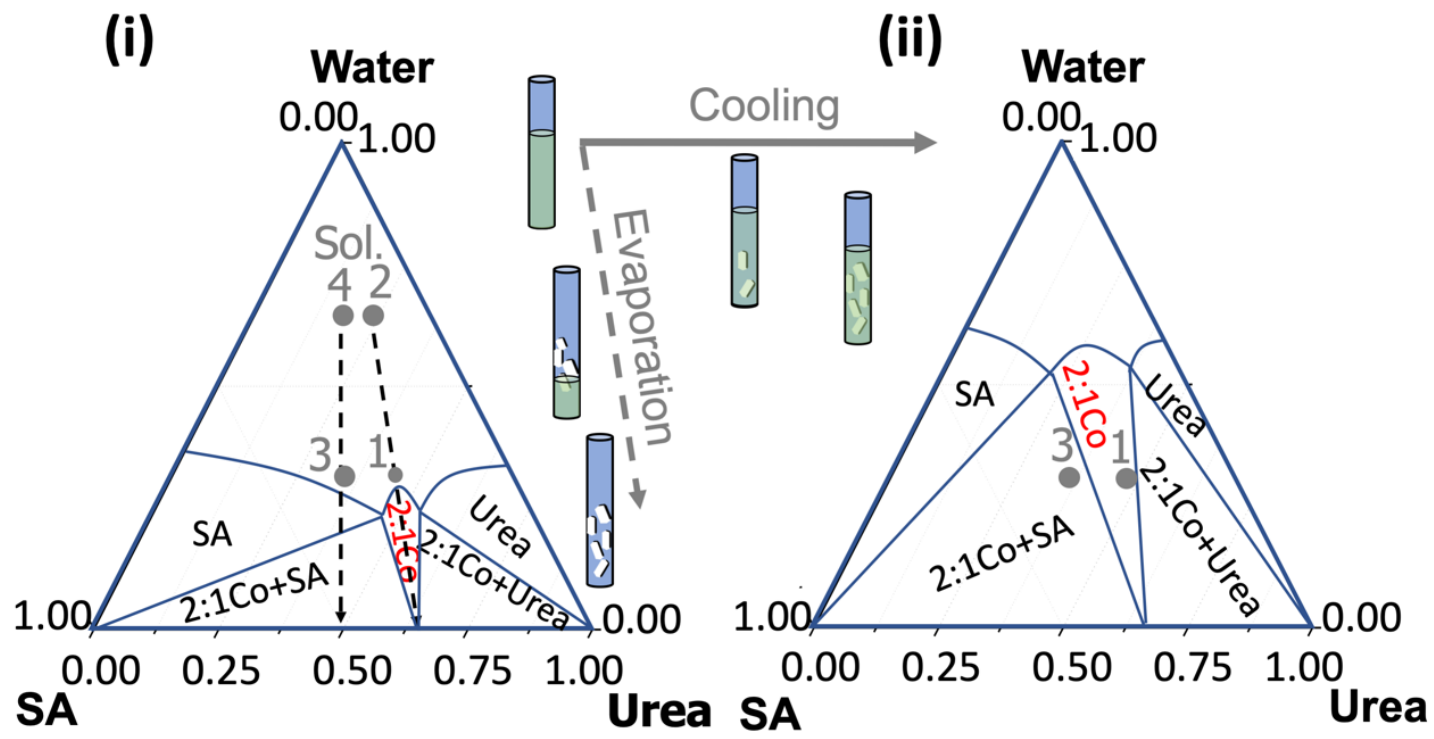

Figure 2. Evaporation and cooling crystallization in schematic water - urea succinic acid ternary phase diagrams, at a high temperature (i) and at a low temperature (ii) of U-SA cocrystals base on the diagram reported at room temperature (Yu et al., 2019).

In the cooling crystallization, CPGs with solutions inside were submerged in 5 $\mathrm{mL}$ hexane solution in a $25 \mathrm{~mL}$ vial at $70^{\circ} \mathrm{C}$. The vial was cooled at $0.2^{\circ} \mathrm{C} / \mathrm{min}$ or $0.8^{\circ} \mathrm{C} / \mathrm{min}$ inside a jacket crystallizer, whose temperature was controlled by a water batch (Tianheng Instrment, THCD-2010), till reaching $30^{\circ} \mathrm{C}$. During the cooling crystallization in Exp 1, 3 and 5, ternary phase diagram changes from 
a high temperature diagram, shown as Figure 2-(i), to a low temperature diagram, shown as Figure 2-(ii), where the composition points in the ternary maintain the same location, but the solubility lines move up (solubility decreases at lowers temperature). Therefore, the composition points in cooling crystallization (in a pure liquid phase at a high temperature) across the solubility lines (at a low temperature), enter and finally locate in liquid and solid phase region.

In the evaporation crystallization, the CPGs with solutions inside were put in a drying oven at 45,55 and $65{ }^{\circ} \mathrm{C}$ under atmosphere pressure for four days. Figure 2-(i) shows the evaporation route of Exp. 2, 4 and 6 (Table 1) in the ternary phase diagram, where the composition points (in pure liquid phase) of the solution in Exp. 2, 4 and 6 move down, across the solubility lines (into liquid and solid phase region), until reach the bottom line, dried into a pure solid phase. The samples of CPGs $(5-10 \mathrm{mg})$ in aluminum pans were scanned by a TA instruments SDT-Q600 (DSC and TG). After cooling and evaporation crystallization, the samples of CPGs $(30-35 \mathrm{mg})$ were analyzed by a X-ray diffractometer (Rigaku smaterlab SE) at room temperature.

Table 1. Crystallization experiments in nano-crystallizer and in bulk solution

\begin{tabular}{|c|c|c|c|c|}
\hline Exp. & Method & Solution scale & [Urea]:[SA] & Cocrystal product \\
\hline & & inside CPG pores* $(\mathrm{nm})$ & & \\
\hline 1 & Cooling & $40-190$ & 2 & 2:1 Co \\
\hline 2 & Evaporation & $40-190$ & 2 & 2:1 Co \\
\hline 3 & Cooling & $40-190$ & 1 & 1:1 \\
\hline 4 & Evaporation & $40,50,60$ & 1 & $1: 1$ \\
\hline & & 100,190 & 1 & 1:1 and $2: 1 \mathrm{Co}$ \\
\hline $\begin{array}{l}5 \\
6\end{array}$ & $\begin{array}{l}\text { Cooling } \\
\text { Evaporation }\end{array}$ & Bulk $^{* *}$ & 2 or 1 & 2:1 Co \\
\hline
\end{tabular}

[]: molar concentration, ${ }^{*}$ mean pore size, ${ }^{* *} 10 \mathrm{~mL}$ solution in $25 \mathrm{~mL}$ vials.

\section{Results}

In Exp. 1 and 2 with solutions of 2:1 concentration ratio between urea and SA, only 2:1 U-SA cocrystals were obtained by both cooling crystallization and 
evaporation crystallization inside CPGs with all pore sizes from 40 to $190 \mathrm{~nm}$ (XRPD shown in Figure 3). The results were consistent with the reference experiments (Exp. 5 and 6) in bulk scale and were in agreement with the literatures that 2:1 cocrystal were stable and always formed with an aqueous solution with 2:1 mole ratio between urea and SA, including cooling crystallization, evaporation crystallization, freeze drying, spray drying, antisolvent crystallization (Alhalaweh et al., 2010).

a) Evaporation crystallization

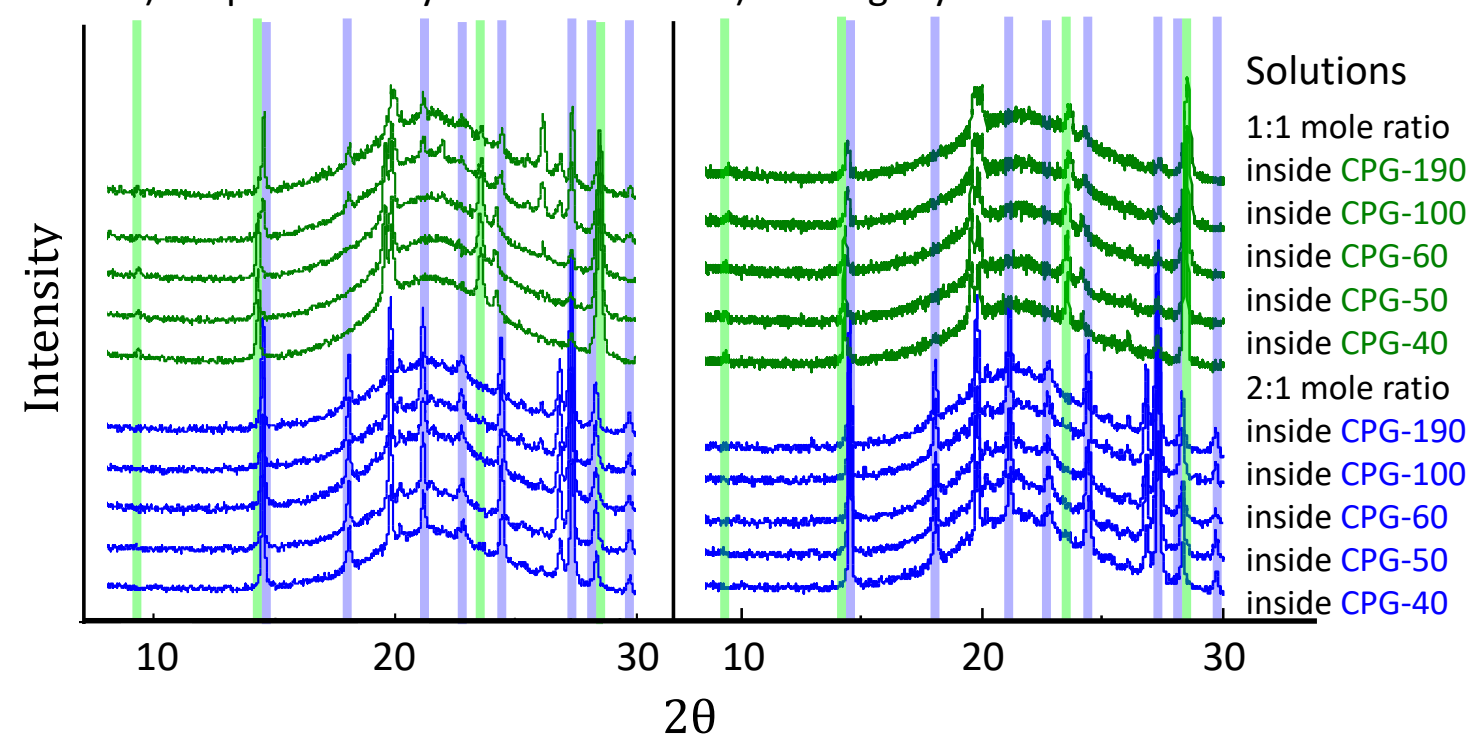

Figure 3 PXRD patterns of cocrystal products by the evaporation and cooling crystallization inside nano-crystallizer in Exp. 1 - 4.

With solutions of 1:1 mole concentration ratio between urea and SA, in Exp 3 by cooling crystallization, only 1:1 U-SA cocrystals (XRPD shown in Figure 3b) were obtained inside CPGs with pore sizes from 40 to $190 \mathrm{~nm}$. To observe the formation of 1:1 cocrystal during the evaporation in Exp 3, solutions in CPGs of $60 \mathrm{~nm}$ with different cooling time were determined by XRPD, however, it was difficult to observe the XRPD peaks until the late stage of the cooling process, due to the influence of the water inside pores of $C P G$ and the small amount of the products crystallized during the cooling process. In Exp 4 by evaporation 
crystallization, only 1:1 U-SA cocrystals were obtained inside CPGs with pore sizes from 40 to $60 \mathrm{~nm}$ (XRPD shown in Figure 3a). To observe the formation of $1: 1$ cocrystal during the evaporation in Exp. 4, solutions in CPGs of $60 \mathrm{~nm}$ with different drying time were determined by XRPD. The peak value increased and half width peak value decreased obviously from $10 \mathrm{~min}$ to $25 \mathrm{~min}$, and after 55 mins there were no obvious changes (supporting information). However, in Exp 4 by evaporation crystallization, 1:1 cocrystals or mixture of 2:1 cocrystals (XRPD shown in Figure 3a) were obtained with CPGs of pore size of 100 and $190 \mathrm{~nm}$ in repeated experiments. In bulk solution, as in Exp. 5 and 6, only 2:1 cocrystals formed in cooling and evaporation crystallization. Since in bulk aqueous solution the 1:1 cocrystals will transform to 2:1 cocrystal (Alhalaweh et al., 2010), no 1:1 cocrystal can coexist in an aqueous solution. However, the final products in Exp. 3 and 4 indicate the coexisting of 1:1 cocrystal in aqueous environment, showing the strong influence of confinement condition on the crystallization process.

To further investigate the evaporation crystallization process in nano-crystallizer, the solution with the 1:1 mole ratio between urea and SA was dropped in the Al-pans, heated up to $55^{\circ} \mathrm{C}$ until totally dried for more than 2 hours. Figure 4 shows that the evaporation speeds in CPGs with different size of pores were similar, i.e. the slopes were similar, and the time for totally drying CPGs with all sizes of pores was about 50 mins, despite a little longer drying time for CPG40. The drying process was consistent with the XRPD of CPGs with solutions during different drying time. Figure 4 proves that in Exp. 4, the evaporation in CPG during about one hour is much slower than the spray-drying, which only takes seconds or even shorter (Alhalaweh et al., 2010). There are not obvious relations between the solution inside the pores with the diameters of the pores, probably due to massive pores are not equally distributed inside CPGs. The slow evaporation process in Exp. 4 also indicated the existing of 1:1 cocrystal in water during a long period. 


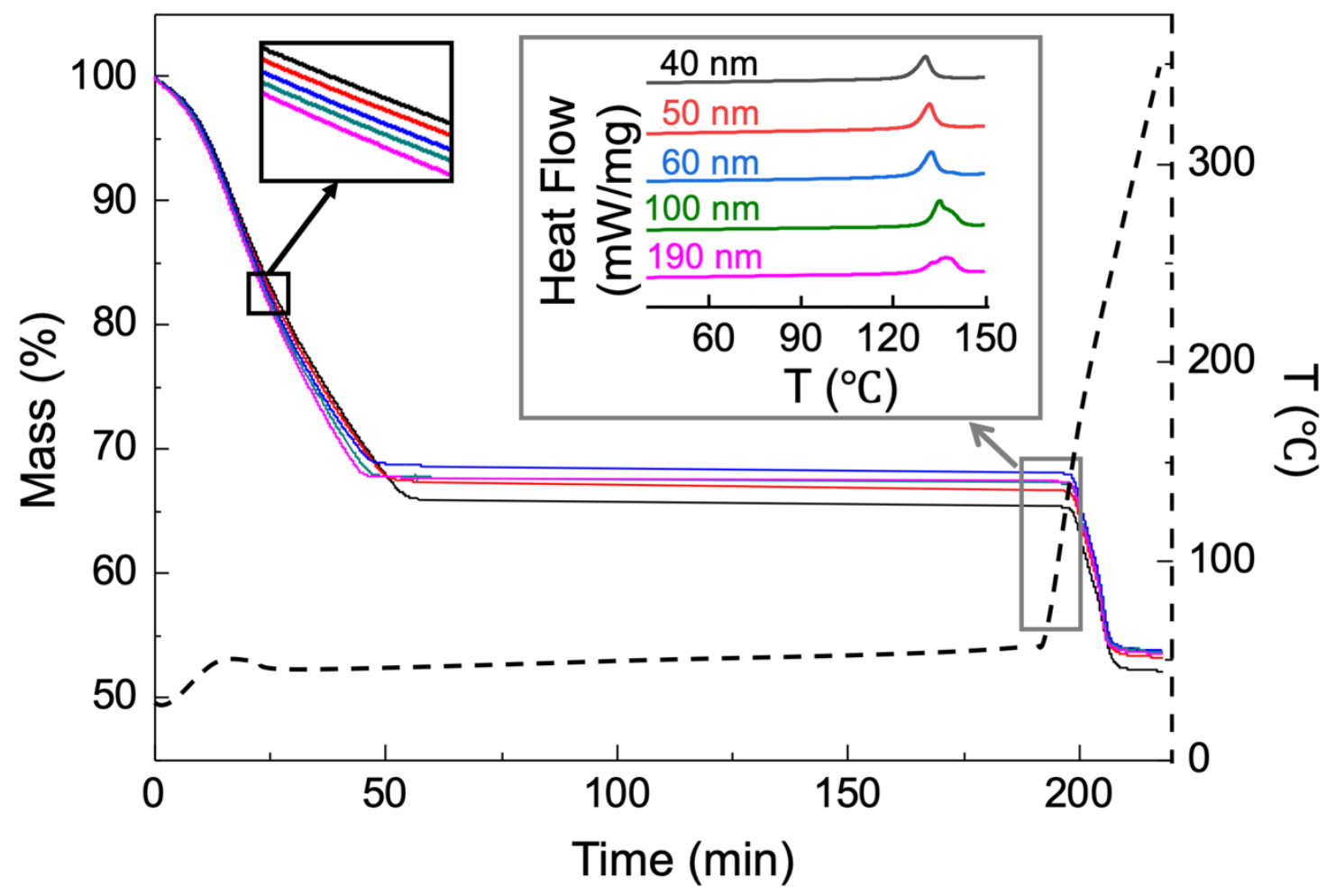

Figure 4. TG and DSC curves of CPG-40, 50, 60, 100 and $190 \mathrm{~nm}$ with solution (as in Exp 4) inside pores.

The dried CPGs in Al pans then heated up to $350{ }^{\circ} \mathrm{C}$, which was above the melting point of bulk $1: 1$ cocrystals $\left(\sim 140{ }^{\circ} \mathrm{C}\right)$ (Alhalaweh et al., 2010$)$ and $2: 1$ U-SA cocrystals $\left(\sim 150^{\circ} \mathrm{C}\right)$. Figure 4 shows one endothermic peak at $130.7^{\circ} \mathrm{C}$, $131.9^{\circ} \mathrm{C}$, and $132.6^{\circ} \mathrm{C}$ for $1: 1 \mathrm{U}-\mathrm{SA}$ cocrystals formed in CPG-40, CPG-50, CPG-60 nm diameter, respectively. The endothermic peak temperatures of 1:1 cocrystals inside CPG with different pore sizes were linearly correlated with the pore diameters (supporting information). The broad peak of the products inside CPG-100 and CPG-190 indicates the final products were not pure 1:1 cocrystal, but composed by the mixture of 1:1 cocrystal and 2:1 cocrystals, which was consistent with the XRPD results in Exp. 4 that the mixtures were obtained within CPG-100 and CPG-190 (supporting information) in evaporation crystallization.

\section{Discussion}


It is reported that the different sizes and shapes of crystals lead to different melting points for same material (Jiang et al., 1999)(Link and El-Sayed, 2000)(Chiarappa et al., 2017). It is difficult to observe the shape of the U-SA cocrystals inside the nano-pores. While, the melting points, in Figure 4, of the $1: 1$ cocrystals increased with the increase in diameter of the nano-crystalilzer used to obtain the cocrystals. Because the pore sizes of CPGs confined the crystal size in nano-crystallizer, the linear dependence (supporting information) between the melting points and the pore sizes, can be due to the linear correlation between the melting point of 1:1 cocrystal and the cocrystal size. The relations were in agreement with literatures that the melting temperature depressed with a decrease in particle size (Jiang and Ward, 2014). The relationship between melting temperature and the size of crystals/particle embedded in CPG pores has been explained by the Gibbs-Thomson Eqn. 1

$$
\Delta T_{m}[\mathrm{~d}]=\frac{4 \gamma_{s-m} M T_{m}}{d \Delta H_{m} \rho_{s}}+T_{m}
$$

where $T_{m}$ is the melting temperature of the bulk crystalline solid, $T_{m}(d)$ is the melting temperature of a confined crystal with diameter $d$ assumed equal to the pore diameter, which is presumed to be equal to the size of the CPG pores, $\Delta \mathrm{H}^{\text {fus }}$ is the molar enthalpy of fusion, $\mathrm{M}$ is the molecular mass, $\rho_{\text {solid }}$ is the density of the solid, $\mathrm{Ys}_{\mathrm{s}-\mathrm{m}}$ is the surface free energy for the solid and melt interface, and $\theta$ is the contact angle between the wall and crystal.

This was the first time to observe the interesting phenomena that the metastable 1:1 U-SA cocrystal maintained stable in an aqueous environment in nano-crystallizer, i.e. the confinement stabilized the metastable 1:1 U-SA cocrystal in aqueous solution. The ternary phase diagram in Figure 2 shows a thermodynamic states of urea, succinic acid and their cocrystals. There is only the 2:1 cocrystal zones and no 1:1 cocrystal zones. The phase diagram chances, due to the influence of the confined conditions. The 1:1 cocrystal zones, which should not exist in the phase diagram due to its instability, appear in the phase diagram. The coexisting of $1: 1$ cocrystal and 2:1 cocrystal zones is also dependent on the size of the nano-crystallizer. This is the first time to observe the nano-crystallizer and the confinement well stabilizes the metastable cocrystal form, but similar phenomena were reported in the 
literatures that the confinement can lead to stabilizations of amorphous and metastable phases of various organic molecules (Wang et al., 2013)(Hamilton et al., 2008)(Rengarajan et al., 2008)(Dwyer et al., 2015) and biominerals (Wang et al., 2014)(Stephens et al., 2010). Therefore, the confinement of nanoscale pores can change the phase diagram and dramatically influence crystallization pathways and crystal polymorphism, as well as preferred orientations of the crystal growth (Nartowski et al., 2015).

(a)

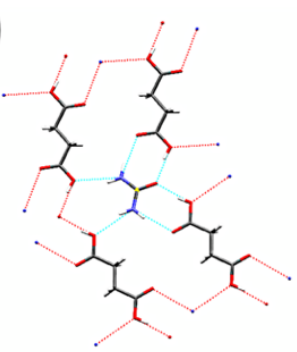

(d)

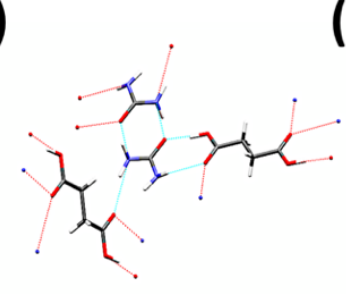

(b)

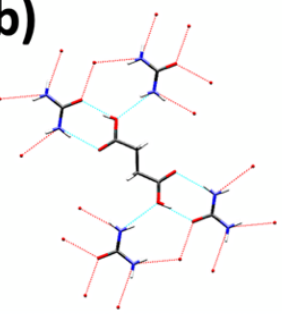

(e)

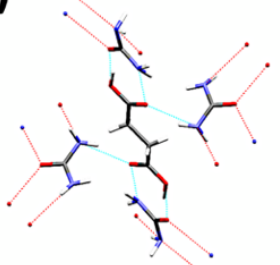

(c)

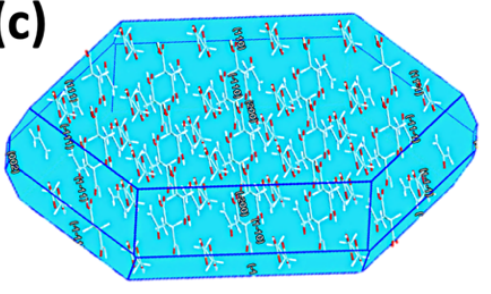

(f)

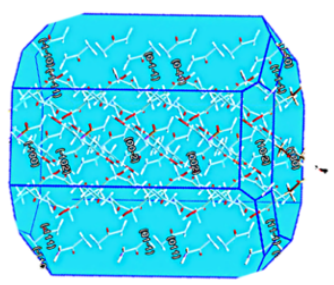

Figure 5. Molecular arrangement of 1:1 U-SA cocrystal, a) and b) and 2:1 cocrystal, c) and d). Hydrogen bonds are indicated as dotted lines. c) and f) is simulated morphology of the 1:1 and 2:1 U-SA cocrystal by BFDH model, respectively.

Comparing with cooling crystallization (Exp 3) with evaporation (Exp 4) with 1:1 mole ration solution, 1:1 cocrystals were obtained within CPG-40, CPG -50 and CPG-60 by both methods. With CPG-100 and CPG-190, cooling crystallization led to $1: 1$ cocrystals, while evaporation crystallization mainly resulted in a mixture of $1: 1$ cocrystal and 2:1 cocrystal in parallel experiments, with occasionally resulted in only 1:1 cocrystals. Three possible reasons are (1) The diameters of the pores. Comparing with the bulk solution, the confinement favored the formation of 1:1 cocrystal, and larger pore size may weaken the influence of the confinement. (2) Confinement by solvent molecules. During the 
drying process, many crystals dried on the surface of the pores without solvent left around the crystals, but during the cooling crystallization, all the crystals formed in the solvent in the confinement environment. The water molecules were also confined in nano-size pores, which may further limit the cocrystal growth directions and spaces. (3) Supersaturation. It is well known that very high supersaturation always tends to nucleate metastable polymorph. During the cooling crystallization, the small volume may have a much higher chance to have larger metastable zone width (Yang and Florence, 2017), driving larger supersaturation at nucleation, which contributed to the formation of metastable crystals $-1: 1$ cocrystals. During the evaporation crystallization, some crystals may form on the surface of the pores due to the residue solution on the wall with decrease volume of the solution, with, correspondingly, low supersaturation at nucleation.

Besides the confinement (Chen et al., 2014), the surface properties may differ the outcome of the metastable polymorph (Yang et al., 2017). Figure 5 shows that in 1:1 cocrystal, each $-\mathrm{NH} 2$ or $-\mathrm{OH}$ bond forms two hydrogen bonds with the molecules besides, however in 2:1 cocrystal half of $-\mathrm{NH} 2$ or $-\mathrm{OH}$ bonds form two hydrogen bonds and half of $-\mathrm{NH} 2$ or $-\mathrm{OH}$ bonds form only one hydrogen bonds with molecules besides. This may indicate 2:1 cocrystals have more chances to form hydrogen bonds with the inside surface of CPG and, therefore, with an increase in specific surface area of the CPGs, i.e. decrease in the pore diameter, nucleation of 2:1 cocrystals was preferably depressed compared with nucleation of $1: 1$ cocrystals. There is no direct correlation revealed between the size of crystal unit cells and the pore size. From the BFDH model, simulated the morphology shows that the 1:1 cocrystals have a larger ratio between length and width, and this may cause preferability of 1:1 cocrystal in the narrow pores, as reported in the literature that the needle shape metastable glycine crystal is favourited in nano-size pores due to its morphology and limited growth direction (Hamilton et al., 2008). The phenomena obvious showed the confinement altered the crystallization process compared with the bulk process, i.e. nucleation of metastable 1:1 cocrystal in cooling and evaporation crystallization, and crystal growth and coexisting of metastable 1:1 cocrystal in an aqueous environment. However, the mechanisms are still not fully understood and may 
be very complicated, and, moreover, it is very difficult to observe the nucleation at a molecular level. It is worth to mention that the metastable 1:1 cocrystal can also be obtained in evaporation of urea and SA in mixture of water and acetonitrile (Alhalaweh et al., 2010), due to the change in solubility, relative stability, and molecular interactions in the solution, which can be the potential reasons for the influence of the confinement. Therefore, the simulations at the molecular level (Giberti et al., 2015)(Salvalaglio et al., 2015) for urea, succinic acid, their cocrystal and solvent molecules should help to better understand the mechanism in the future investment.

\section{Conclusions}

In the bulk solution, the stable 2:1 urea-succinic acid cocrystal formed by the cooling and evaporation crystallization, independent on the mole ratio of urea and succinic acid in the solution. With the 2:1 urea-succinic acid solution, the 2:1 cocrystals were obtained inside CPGs, in agreement with the cocrystallization in the bulk solution. However, in this work, it was the first time to observe the formation of the metastable 1:1 urea-succinic acid cocrystal in an aqueous solution by the slow cooling and evaporation inside the nanocrystallizer / nano-pores of CPG. DSC and TGA proved that the 1:1 cocrystals coexisted in the aqueous solution inside the nano-pores during the evaporation, while in a bulk solution the $1: 1$ cocrystal will rapidly transform to the stable $2: 1$ cocrystals. With the $1: 1$ urea-succinic acid solution in the small pores below 60 $\mathrm{nm}$, pure 1:1 cocrystal products crystallized, whose melting points were lower within the smaller pores. With the solutions in the pores above $100 \mathrm{~nm}$, mixtures of $1: 1$ and $2: 1$ cocrystal formed. The confinements altered the cocrystallization process, leading to different cocrystal products with different properties, compared with the cocrystallization process in the bulk solution. The simulations at the molecular level for the cocrystal systems can be helpful to further understand the mechanism.

\section{Acknowledgements}


This study was supported by National Science Foundation (NSFC. 21978234), Natural Science Foundation of Shaanxi Province (2013JQ2020), and HY is grateful to EP/T005378/1 for the support.

\section{References}

Alhalaweh, A., George, S., Boström, D., Velaga, S.P., 2010. 1: 1 and 2: 1 Ureasuccinic acid cocrystals: structural diversity, solution chemistry, and thermodynamic stability. Cryst. Growth Des. 10, 4847-4855.

Charnay, C., Bégu, S., Tourné-Péteilh, C., Nicole, L., Lerner, D.A., Devoisselle, J.-M., 2004. Inclusion of ibuprofen in mesoporous templated silica: drug loading and release property. Eur. J. Pharm. Biopharm. 57, 533-540.

Chen, W., Park, S.J., Kong, F., Li, X., Yang, H., Heng, J.Y.Y., 2019. High ProteinLoading Silica Template for Heterogeneous Protein Crystallization. Cryst. Growth Des. 20, 866-873.

Chen, Y., Li, L., Yao, J., Ma, Y.-Y., Chen, J.-M., Lu, T.-B., 2016. Improving the solubility and bioavailability of apixaban via apixaban-oxalic acid cocrystal. Cryst. Growth Des. 16, 2923-2930.

Chen, Y., Wang, S., Ye, J., Li, D., Liu, Z., Wu, X., 2014. Insights into the effect of nanoconfinement on molecular interactions. Nanoscale 6, 9563-9567.

Chiarappa, G., Piccolo, A., Colombo, I., Hasa, D., Voinovich, D., Moneghini, M., Grassi, G., Farra, R., Abrami, M., Posocco, P., 2017. Exploring the shape influence on melting temperature, enthalpy, and solubility of organic drug nanocrystals by a thermodynamic model. Cryst. Growth Des. 17, 40724083.

Coceani, N., Magarotto, L., Ceschia, D., Colombo, I., Grassi, M., 2012. Theoretical and experimental analysis of drug release from an ensemble of polymeric particles containing amorphous and nano-crystalline drug. Chem. Eng. Sci. 71, 345-355.

Dai, X.-L., Chen, J.-M., Lu, T.-B., 2018. Pharmaceutical cocrystallization: an effective approach to modulate the physicochemical properties of solidstate drugs. CrystEngComm 20, 5292-5316.

Dosseh, G., Xia, Y., Alba-Simionesco, C., 2003. Cyclohexane and benzene confined in MCM-41 and SBA-15: confinement effects on freezing and melting. J. Phys. Chem. B 107, 6445-6453.

Duggirala, N.K., Perry, M.L., Almarsson, Ö., Zaworotko, M.J., 2016. Pharmaceutical cocrystals: along the path to improved medicines. Chem. Commun. 52, 640-655.

Duggirala, N.K., Smith, A.J., Wojtas, Ł., Shytle, R.D., Zaworotko, M.J., 2014. Physical stability enhancement and pharmacokinetics of a lithium ionic cocrystal with glucose. Cryst. Growth Des. 14, 6135-6142.

Dwyer, L.M., Michaelis, V.K., O'Mahony, M., Griffin, R.G., Myerson, A.S., 2015. Confined crystallization of fenofibrate in nanoporous silica. 
CrystEngComm 17, 7922-7929.

Giberti, F., Salvalaglio, M., Mazzotti, M., Parrinello, M., 2015. Insight into the nucleation of urea crystals from the melt. Chem. Eng. Sci. 121, 51-59.

Gopi, S.P., Banik, M., Desiraju, G.R., 2016. New cocrystals of hydrochlorothiazide: optimizing solubility and membrane diffusivity. Cryst. Growth Des. 17, 308-316.

Ha, J.-M., Hamilton, B.D., Hillmyer, M.A., Ward, M.D., 2009. Phase behavior and polymorphism of organic crystals confined within nanoscale chambers. Cryst. Growth Des. 9, 4766-4777.

Ha, J.-M., Wolf, J.H., Hillmyer, M.A., Ward, M.D., 2004. Polymorph selectivity under nanoscopic confinement. J. Am. Chem. Soc. 126, 3382-3383.

Hamilton, B.D., Hillmyer, M.A., Ward, M.D., 2008. Glycine polymorphism in nanoscale crystallization chambers. Cryst. Growth Des. 8, 3368-3375.

Jackson, C.L., McKenna, G.B., 1996. Vitrification and crystallization of organic liquids confined to nanoscale pores. Chem. Mater. 8, 2128-2137.

James, S.L., Adams, C.J., Bolm, C., Braga, D., Collier, P., Friščić, T., Grepioni, F., Harris, K.D.M., Hyett, G., Jones, W., 2012. Mechanochemistry: opportunities for new and cleaner synthesis. Chem. Soc. Rev. 41, 413-447.

Jiang, Q., Hu, C., Ward, M.D., 2013. Stereochemical control of polymorph transitions in nanoscale reactors. J. Am. Chem. Soc. 135, 2144-2147.

Jiang, Q., Shi, H.X., Zhao, M., 1999. Melting thermodynamics of organic nanocrystals. J. Chem. Phys. 111, 2176-2180.

Jiang, Q., Ward, M.D., 2014. Crystallization under nanoscale confinement. Chem. Soc. Rev. 43, 2066-2079.

Kanakubo, M., Hiejima, Y., Minami, K., Aizawa, T., Nanjo, H., 2006. Melting point depression of ionic liquids confined in nanospaces. Chem. Commun. 1828-1830.

Kaur, R., Cavanagh, K.L., Rodríguez-Hornedo, N., Matzger, A.J., 2017. Multidrug cocrystal of anticonvulsants: influence of strong intermolecular interactions on physiochemical properties. Cryst. Growth Des. 17, 50125016.

Link, S., El-Sayed, M.A., 2000. Shape and size dependence of radiative, nonradiative and photothermal properties of gold nanocrystals. Int. Rev. Phys. Chem. 19, 409-453.

Munekawa, Y., Oaki, Y., Sato, K., Imai, H., 2015. Incorporation of organic crystals into the interspace of oriented nanocrystals: morphologies and properties. Nanoscale 7, 3466-3473.

Nartowski, K.P., Tedder, J., Braun, D.E., Fábián, L., Khimyak, Y.Z., 2015. Building solids inside nano-space: from confined amorphous through confined solvate to confined 'metastable'polymorph. Phys. Chem. Chem. Phys. 17, 24761-24773.

Nelson, P.S., 1995. Method for labeling the 3'terminus of a synthetic oligonucleotide using a unique multifunctional controlled pore glass (MF$\mathrm{CPG}$ ) reagent in solid phase oligonucleotide synthesis. 
Padrela, L., Rodrigues, M.A., Tiago, J., Velaga, S.P., Matos, H.A., de Azevedo, E.G., 2014. Tuning physicochemical properties of theophylline by cocrystallization using the supercritical fluid enhanced atomization technique. J. Supercrit. Fluids 86, 129-136.

Powell, K.A., Croker, D.M., Rielly, C.D., Nagy, Z.K., 2016. PAT-based design of agrochemical co-crystallization processes: A case-study for the selective crystallization of $1: 1$ and $3: 2$ co-crystals of $p$ toluenesulfonamide/triphenylphosphine oxide. Chem. Eng. Sci. 152, 95108.

Rengarajan, G.T., Enke, D., Steinhart, M., Beiner, M., 2008. Stabilization of the amorphous state of pharmaceuticals in nanopores. J. Mater. Chem. 18, 2537-2539.

Salvalaglio, M., Perego, C., Giberti, F., Mazzotti, M., Parrinello, M., 2015. Molecular-dynamics simulations of urea nucleation from aqueous solution. Proc. Natl. Acad. Sci. 112, E6-E14.

Scherer, G.W., 1999. Crystallization in pores. Cem. Concr. Res. 29, 1347-1358. Skorupska, E., Jeziorna, A., Paluch, P., Potrzebowski, M.J., 2014. Ibuprofen in mesopores of mobil crystalline material 41 (MCM-41): a deeper understanding. Mol. Pharm. 11, 1512-1519.

Stephens, C.J., Ladden, S.F., Meldrum, F.C., Christenson, H.K., 2010. Amorphous calcium carbonate is stabilized in confinement. Adv. Funct. Mater. 20, 2108-2115.

Tiago, J.M., Padrela, L., Rodrigues, M.A., Matos, H.A., Almeida, A.J., Azevedo, E.G. de, 2013. Single-step co-crystallization and lipid dispersion by supercritical enhanced atomization. Cryst. Growth Des. 13, 4940-4947.

Vekilov, P.G., 2010. The two-step mechanism of nucleation of crystals in solution. Nanoscale 2, 2346-2357.

Wang, Y.-W., Christenson, H.K., Meldrum, F.C., 2014. Confinement increases the lifetimes of hydroxyapatite precursors. Chem. Mater. 26, 5830-5838.

Wang, Y., Christenson, H.K., Meldrum, F.C., 2013. Confinement leads to control over calcium sulfate polymorph. Adv. Funct. Mater. 23, 5615-5623.

Wiedenfeld, H., Knoch, F., 1990. Urine Complexes With Dicarboxylic-Acids. 1. Structure of Succinic Acid Urea. ACTA Crystallogr. Sect. C-CRYSTAL Struct. Commun. 46, 1038-1040.

Yang, H., Florence, A.J., 2017. Relating induction time and metastable zone width. CrystEngComm 19, 3966-3978.

Yang, H., Song, C.L., Lim, Y.X.S., Chen, W., Heng, J.Y.Y., 2017. Selective crystallisation of carbamazepine polymorphs on surfaces with differing properties. CrystEngComm 19, 6573-6578.

Yang, X., Ong, T.-C., Michaelis, V.K., Heng, S., Griffin, R.G., Myerson, A.S., 2015. Formation of organic molecular nanocrystals under soft confinement. CrystEngComm 17, 6044-6052.

Yao, G.-B., Wang, L., Sun, Y.-P., Yi, J.-K., Meng, L., Zhao, H.-K., 2014. Ternary phase diagram for systems of succinic acid+ urea+ water, glutaric acid+ 
urea+ water, and adipic acid+ urea+ water at (288.15 and 303.15) K. J. Chem. Eng. Data 59, 4081-4089.

Yu, Q., Pu, J., Qi, M., Yang, H., 2019. Jumping into Metastable 1: 1 UreaSuccinic Acid Cocrystal Zone by Freeze-Drying. Cryst. Growth Des. 19, 1505-1508.

Zabara, A., Meikle, T.G., Newman, J., Peat, T.S., Conn, C.E., Drummond, C.J., 2017. The nanoscience behind the art of in-meso crystallization of membrane proteins. Nanoscale 9, 754-763. 